\title{
Advancement and Trend of Nondispersive Infrared Carbon Dioxide Sensor
}

\author{
Shuqing Han ${ }^{1,2}$, Jiajia Liü, 2, Mengshuai Zhür, \\ Chen Shen ${ }^{1,2}$, and Jianhua Zhang ${ }^{1,2, a}$ \\ ${ }^{1}$ Agricultural Information Institute of Chinese Academy of Agricultural Sciences, Beijing, 100081, \\ China \\ ${ }^{2}$ Key Laboratory of Agri-information Services Technology, Ministry of Agriculture, Beijing, 100081, \\ China \\ a Corresponding author, email: zhangjianhua@caas.cn
}

Keywords: Carbon dioxide, NDIR, and gas sensor

\begin{abstract}
This paper briefly introduces measurement principles of Nondispersive Infrared (NDIR) carbon dioxide $\left(\mathrm{CO}_{2}\right)$ sensors as well as summarizes performance characteristics of NDIR $\mathrm{CO}_{2}$ sensors, factors affecting the accuracy, calibration methods, market situations and trend of NDIR $\mathrm{CO}_{2}$ sensors.
\end{abstract}

\section{Introduction}

Carbon Dioxide $\left(\mathrm{CO}_{2}\right)$ is one of the important components in the atmosphere. $\mathrm{CO}_{2}$ is used in crop growth, animal husbandry production, agricultural product storage, food processing, environmental monitoring, industrial production, and other fields. It is also an important greenhouse gas. Therefore, to promote the development of national economy, it is very important to improve the level of $\mathrm{CO}_{2}$ monitoring and control in China. Currently, $\mathrm{CO}_{2}$ sensors have been applied widely in agriculture, aquaculture, food processing, industry, and other fields. In facility agriculture, $\mathrm{CO}_{2}$ sensors are used to monitor changes of $\mathrm{CO}_{2}$ concentration in greenhouse. When the $\mathrm{CO}_{2}$ concentration is low, the increase of $\mathrm{CO}_{2}$ can improve the efficiency of plant photosynthesis as well as the quality and yield of agricultural products. In animal husbandry production, the high concentration of $\mathrm{CO}_{2}$ will lead to the decline of animal appetite and immunity as well as impact on the animal production capacity even though $\mathrm{CO}_{2}$ is not toxic. When the concentration of $\mathrm{CO}_{2}$ is too high in monitoring, ventilation will do timely to improve the air quality of livestock and poultry house, and increase the production efficiency of livestock and poultry.

$\mathrm{CO}_{2}$ detection methods mainly include gas chromatography, titration method, solid electrolyte sensor, electrochemical, capacitive, optical fiber detection method, and detection method based on non-dispersive infrared (NDIR) [1]. NDIR $\mathrm{CO}_{2}$ sensors have high sensitivity, good selectivity, good stability, long service life, low environmental impact, high response speed, non-toxicity, easy installation, perfect reproducibility and good repeatability, and other advantages. NDIR $\mathrm{CO}_{2}$ sensors are the most mature sensors and have been widely used. However, there are still several problems with NDIR $\mathrm{CO}_{2}$ sensors, for example, the optical characteristics of infrared light source will change, high cost, difficult miniaturization and others. On the basis of introducing the working principle of NDIR $\mathrm{CO}_{2}$ sensors, the paper summarizes performance characteristics of NDIR $\mathrm{CO}_{2}$ sensors, the factors affecting the accuracy of NDIR $\mathrm{CO}_{2}$ sensors, the calibration method of NDIR $\mathrm{CO}_{2}$ sensors, and the market situation, research status and development trend of NDIR $\mathrm{CO}_{2}$ sensors. 


\section{Principles of NDIR $\mathrm{CO}_{2}$ Sensor}

$\mathrm{CO}_{2}$ absorbs infrared that the wavelength is $4.26 \mathrm{um}$. When infrared light is used to irradiate any gas with $\mathrm{CO}_{2}, \mathrm{CO}_{2}$ will absorb some infrared. The light intensity through infrared is related to the concentration of $\mathrm{CO}_{2}$, and the absorption of $\mathrm{CO}_{2}$ to infrared can meet the law of Lamber Beer. According to the law of Lamber Beer, the emission light intensity is shown as follow:

$$
I=I_{0} \exp (-\mathrm{KCL})
$$

Among them, $I_{0}$ is the incident light intensity, $I$ is the transmitted light intensity, $\mathrm{K}$ is the gas absorption coefficient that is related to gas type, spectral wavelength, pressure, temperature and other factors. $C$ is the concentrated of gas to be measured, and $L$ is the length of gas chamber.

A NDIR $\mathrm{CO}_{2}$ sensor is composed generally of infrared light source, gas measurement chamber, optical filter, dual channel thermopile sensor, filter, small signal amplification circuit, and others as shown in Fig.1. The gas enters from the inlet and gets out from the outlet. Infrared light source emits infrared intermittently to penetrate measured gas and optical filter. Dual channel thermopile sensor is used to measure the sensitive wavelength of $\mathrm{CO}_{2}$ and the light intensity of reference wavelength. The output voltage of thermopile sensor is weak relatively. It is necessary for filtering weak signals to remove noises and amplify signals. Infrared from infrared light source passes through measured gas, and the light that the wavelength is $4.26 \mathrm{um}$ will be absorbed by $\mathrm{CO}_{2}$, and the light intensity will become weak. However for another reference light, the light intensity is not changed because the light is not absorbed by gas. The concentration of measured gas will be calculated by measuring the absorption of light that the wavelength is $4.26 \mathrm{um}$.

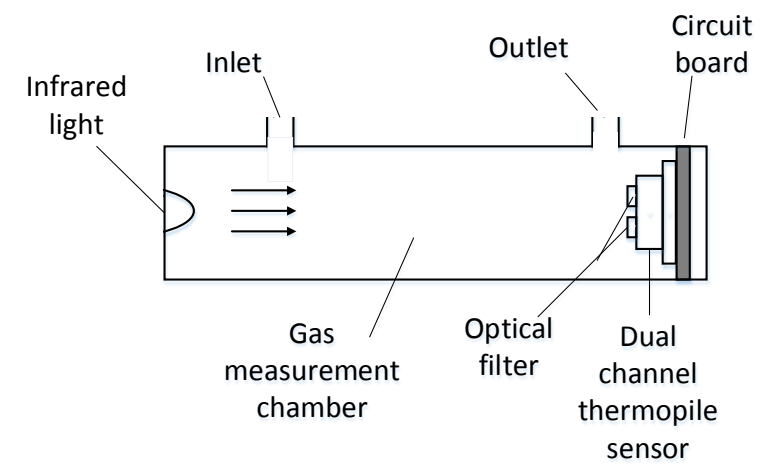

Fig.1. Schematic Diagram of NDIR $\mathrm{CO}_{2}$ Sensor

\section{Performance Characteristics of $\mathrm{CO}_{2}$ Sensor}

Performance characteristics measuring whether NDIR $\mathrm{CO}_{2}$ sensors are good or poor mainly include accuracy, measuring range, sensitivity, selectivity, response time, stability, power consumption, and maintenance convenience.

Accuracy: the error of the sensor indicating value and the actual value.

Measuring range: the maximum and minimum possible value that the sensor can measure.

Sensitivity: the minimum concentration of $\mathrm{CO}_{2}$ that can be detected.

Selectivity: the ability that the concentration of $\mathrm{CO}_{2}$ can be detected in mixed gas.

Response time: the necessary time that sensor can sense the change when there is any concentration change of $\mathrm{CO}_{2}$.

Stability: the time to carry out stable measurement.

Power consumption: the effect of power consumption and sensor self-heating on measurement accuracy.

Maintenance convenience: the specified calibration interval and the calibration service to be used. 


\section{Factors Affecting the Accuracy of NDIR $\mathrm{CO}_{2}$ Sensor}

Factors affecting the accuracy of NDIR $\mathrm{CO}_{2}$ sensor include temperature, atmospheric pressure, dust, light source characteristics drift, and so on [2]. Actually, the working principle of NDIR is to measure the concentration of gas inside the measurement chamber. Therefore, both external environment temperature and atmospheric pressure will affect the measurement results.

Temperature: thermopile sensors are easy to be affected by temperature, which leads to the decrease of the accuracy of $\mathrm{CO}_{2}$ sensor [3].

Atmospheric pressure will affect the absorption of $\mathrm{CO}_{2}$ to infrared so that the measurement value of $\mathrm{CO}_{2}$ sensor produces fluctuations [4].

For a long period, dust will be deposited in the gas chamber and on filter surface. It will affect the measurement accuracy of sensors.

Water vapor will affect the measurement accuracy of sensor and even pollute the optical path to damage sensor in condensation.

For characteristic drift, the light source based on NDIR $\mathrm{CO}_{2}$ sensor is an incandescent lamp. Current passes through filament, can lead to a high temperature of filament and then give out light. During the glowing process, some atoms on filament evaporate. After cooling, most of atoms will be absorbed again on filament, but some atoms will precipitate on the inner wall of bulb glass. As time goes by, filament will become thinner and thinner, and the light emitted by incandescent lamp will become darker and darker. Not only so, that part of metal atoms absorbed again on filament will affect the emission spectra of incandescent lamp, which will eventually affect the intensity of infrared light and the measurement of $\mathrm{CO}_{2}$.

\section{Calibration Methods for NDIR $\mathrm{CO}_{2}$ Sensor}

Calibration methods of NDIR $\mathrm{CO}_{2}$ sensor mainly include nitrogen calibration, chemical method calibration, automatic background calibration, and automatic environment long-term calibration.

Nitrogen calibration means to make nitrogen as standard gas, and fill nitrogen into closed chamber to calibrate the zero of sensors [5].

Chemical method calibration means to pump air into soda lime to obtain a kind of gas without $\mathrm{CO}_{2}$ after chemical reactions, and calibrate the zero of sensors with the gas.

Automatic background calibration means to calibrate with a characteristics that the outdoor concentration of $\mathrm{CO}_{2}$ is about $400 \mathrm{ppm}$ normally. Man is the main source of $\mathrm{CO}_{2}$ in room. The concentration of indoor $\mathrm{CO}_{2}$ will be consistent with that of outdoor $\mathrm{CO}_{2}$ after there is not any individual activity for $4 \sim 8$ hours. The method of automatic background calibration is to record the minimum concentration of $\mathrm{CO}_{2}$ with internal microprocessor in sensor within 24 hours a day. Assuming that the minimum concentration of $\mathrm{CO}_{2}$ shall be consistent with that of outdoor $\mathrm{CO}_{2}$, the sensor can be calibrated.

Reference channel calibration means to select the waveband that is not affected by $\mathrm{CO}_{2}$ as a reference channel. Sensor reads the measurement value of reference channel every day. Referring to the change of channel measurement value means the change of optical path for sensor. The change of optical path can lead to the drift of sensor. Sensor calibrates and measures channels automatically to prevent any drift.

\section{Market Status of NDIR Sensor}

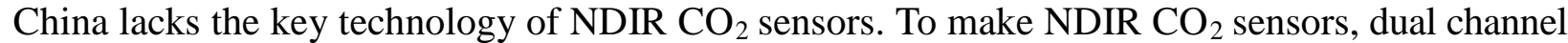
thermopile sensors need to be imported from foreign country. At present, major manufacturers of thermopile sensors mainly include TI, PerkinElmer, MEAS, Hamamatsu, SEMITEC, HEIMANN, Melexis, OmniWise, and others. Among them, the companies which manufacture dual channel thermopile sensors include HEIMANN, Hamamatsu and OminiWise. China should increase the investment in the research and development of sensor, acquire key technology and get rid of the dependence of foreign parts. 


\section{Current researches of NDIR Sensor}

In order to solve the problem that the stability of $\mathrm{CO}_{2}$ sensor will be declined with the passage of time, Jacob Y Wong had proposed an absorption Biased NDIR measurement method [8]. The measurement gas chamber of NDIR sensor has been redesigned with the method to increase the measurement channel of reference signal. However, the length of measurement channel for reference signal is different from that of measurement channel for measured signal. Detectors of measured and reference signal are equipped with the same narrow-band light filters that center wavelength (CWL), full width at half maximum (FWHM) and transmittance are same. In the method, the deviation of $\mathrm{CO}_{2}$ absorbed from two measurement channels is used to calculate the concentration of measured gas. Two measurement channels share a measurement gas chamber with same temperature, same light filter, and same thermopile sensor. When external environment temperature is changed or optical devices are aging, the specific value of two measurement channels will not be affected. Therefore, the stability of NDIR sensor for absorption deviation has been improved.

$\mathrm{Xu}$ Wang, et al [10] have proposed a method of pressure regulation and designed the prototype of pressure regulation NDIR sensor in order to improve the sensitivity of NDIR sensor and eliminate the zero drift caused by optical device aging. The pressure regulation NDIR sensor is composed of pressure adjustment module, infrared signal measurement module, and signal analysis module. Test results show that the sensitivity of sensor has been increased by 8.6 times when the pressure is 900 $\mathrm{kPa}$, and the zero drift can be eliminated by calculating the measured values under two different pressures.

Vaisala Corporation had developed the next generation of infrared light source technology Microglow to improve the service life of infrared light source greatly, shorten the starting time of light source, and reduce the power consumption. The power consumption is only $25 \%$ of original infrared light source, and the service life has been extended by $50 \%$.

In the application research, T.A.Vincent [9] had developed a sensor monitoring $\mathrm{CO}_{2}$ from patient based on NDIR principle in order to reduce the cost of $\mathrm{CO}_{2}$ sensor in clinical application. The sensor is composed of a wide range of infrared light source (CCS102, Cambridge CMOS Sensors Ltd, UK), thermocouple sensor (J21, Heimann Sensor, Germany), 4.3 um light filter (bandwidth $180 \mathrm{~nm}$ ), and phase-locked amplifier circuit. The sensor has advantages such as low cost, small volume, short response time, and low power consumption as well as can operate stably under the condition of high humidity.

$\mathrm{CO}_{2}$ sensor in greenhouse needs to overcome the influence of high temperature and humidity environment. However, most of existing $\mathrm{CO}_{2}$ sensors are for the industrial environment, Zhang Xin et al has redesigned the $\mathrm{CO}_{2}$ sensor based on NDIR principle to improve the environmental adaptability problems of existing $\mathrm{CO}_{2}$ sensor in agricultural applications, and further reduce the power consumption of $\mathrm{CO}_{2}$ sensor. The sensor has been equipped with the latest low-power-consumption sensor manufactured by UK GSS, increased with control modules and remote communication modules, added waterproof and breathable materials outside of sensor, and reduced the effect of water vapor on optical sensor path. It had also been integrated with 4 kinds of calibration modes such as outdoor air calibration, environment long-term calibration, zero calibration, and reference point calibration. Compared with traditional sensors after testing, the design has significant advantages in sensor waterproofness, accuracy compensation, calibration mode, energy consumption management, and other aspects. These had extended the service life and calibration period of $\mathrm{CO}_{2}$ sensor significantly in greenhouse environment, and have a good application prospect.

\section{Conclusions}

The ideal NDIR $\mathrm{CO}_{2}$ sensor shall achieve the goals of low power consumption, low cost, small volume, little annual drift, good stability and easy maintenance. In order to realize the miniaturization, it is necessary to design a more compact gas measurement chamber [6]. To improve 
the sensitivity, it is necessary to increase the optical path. In order to increase the system signal-to-noise ratio and stability, it is necessary to allow more light reach the detector through gas chamber. In order to reduce the annual drift of sensor, it is necessary to research and develop new light sources, and improve the stability of light source and the anti-ageing ability. In order to maintain sensor easily, it is necessary to research and develop technologies which can calibrate sensors at anytime and anywhere [7]. In order to improve the stability of sensor and reduce the annual drift, it is necessary to optimize, design, and measure gas chambers [8]. At the same time, it is necessary to select anti-corrosion, dust-proof, and anti-pollution materials to make gas chambers.

\section{Acknowledgement}

In this paper, the research was sponsored by the Incremental Budget Project of Fundamental Research Funds for CAAS "Research and Development and Application on Key Technology and Equipment of Agricultural Product Market Information Acquisition” (Project No. 2015ZL018) and the National Natural Science Foundation of China (Grant No. 31501229).

\section{References}

[1] Yuan Chao, He Baoshan, Han Xiaoxian, et al. Research Progress on Detection of Carbon Dioxide Gas. Jiangxi Journal of Agriculture, 2009, 21:133-136. DOI:doi:10.3969/j.issn.1001-8581.2009.06.044.

[2] Marinov M, Nikolov G, Gieva E, et al. Improvement of NDIR Carbon Dioxide Sensor Accuracy[J].

[3] Park J S, Cho H C, Yi S H. NDIR $\mathrm{CO}_{2}$ gas sensor with improved temperature compensation[J]. Procedia Engineering, 2010, 5(6):303-306.

[4]Wang X, Rödjegård H, Oelmann B, et al. High performance CO2 measurement based on pressure modulation[J]. Procedia Engineering, 2010, 5: 1208-1211.

[5] Zhang Xin, Guo Rui, Zheng Wengang, et al. Research on Special Low Power Consumption Wireless $\mathrm{CO}_{2}$ Sensor and Calibration Method in Greenhouse [J]. Xinjiang Agricultural Sciences, 2014,51:1028-1036.

[6] Wong J Y. Intrinsically safe NDIR gas sensor in a can: U.S. Patent 8,415,626[P]. 2013-4-9.

[7] Wong, Jacob Y. Re-calibration methodology for NDIR gas sensors: US, doi:US8178832 B1[P]. 2012.

[8] Wong, Jacob Y. Absorption biased NDIR gas sensing methodology: US, doi:US8143581 B2[P]. 2012.

[9] Vincent T.A., Urasinska-Wojcik B., Gardner J.W., Development of a Low-cost NDIR System for ppm Detection of Carbon Dioxide in Exhaled Breath Analysis[J], Procedia Engineering, 2015, 120: 388-391

[10] Xu W, Rödjegård $\mathrm{H}$, Oelmann $\mathrm{B}$, et al. High performance $\mathrm{CO}_{2}$ measurement based on pressure modulation[J]. Procedia Engineering, 2010, 5(5):1208-1211. 